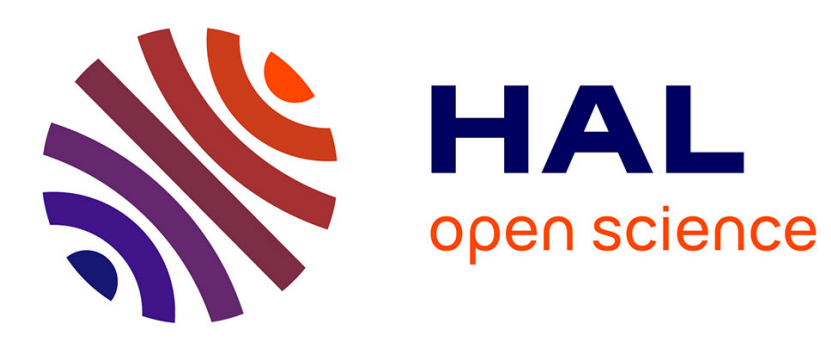

\title{
De l'intermédiation à la prescription : le cas de la télévision
}

\author{
P. J. Benghozi, Thomas Paris
}

\section{To cite this version:}

P. J. Benghozi, Thomas Paris. De l'intermédiation à la prescription: le cas de la télévision. Revue Française de Gestion, 2003, 1 (142), pp.205-227. hal-00231010

\section{HAL Id: hal-00231010 https://hal.science/hal-00231010}

Submitted on 31 Jan 2008

HAL is a multi-disciplinary open access archive for the deposit and dissemination of scientific research documents, whether they are published or not. The documents may come from teaching and research institutions in France or abroad, or from public or private research centers.
L'archive ouverte pluridisciplinaire HAL, est destinée au dépôt et à la diffusion de documents scientifiques de niveau recherche, publiés ou non, émanant des établissements d'enseignement et de recherche français ou étrangers, des laboratoires publics ou privés. 


\title{
DE L'INTERMEDIATION A LA PRESCRIPTION : \\ LE CAS DE L'AUDIOVISUEL
}

\author{
Pieme-J ean BeNGHOZ \& Thomas PARIS
}

Article paru dans la Revue Française de Gestion

Vol 29 - numéro 402 - janvier/ février 2003 - pp 205-227

Pierre-Jean BENGHOZI

directeur de recherche au CNRS CRG École polytechnique

benghozi@poly.polytechnique.fr
Thomas PARIS

Chargé de recherche CNRS EURISTIK / CRG École polytechnique

thomas.paris@polytechnique.fr 


\section{Introduction}

Depuis plusieurs dizaines d'années, l'industrie de l'audiovisuel a profondément évolué dans ses modèles d'affaires : le téléspectateur est passé d'un statut d'usager à une position de consommateur potentiel, le modèle de la chaîne hertzienne généraliste autonome en oligopole s'est estompé au profit de chaînes à péage et de bouquets de chaînes pour le câble et le satellite. Ces étapes ont donné lieu à une multiplication sensible du nombre de chaînes, à l'évolution de leur programmation; elles marquent, plus profondément, l'évolution de la chaîne de valeur et de la structuration du marché de la télévision. Le cas est particulièrement intéressant dans la mesure où il est illustratif d'une économie de la prescription mal étudiée dans la littérature de gestion comme dans les travaux des économistes. Le phénomène de la prescription, bien identifié des chercheurs et praticiens du marketing, s’observe dans de nombreux secteurs : médecins prescripteurs de médicaments, enseignants prescripteurs d'ouvrages scolaires, architectes ou maîtres d'œuvre, conseillers financiers et gestionnaires de patrimoine... Comme on le verra plus précisément sur le cas de l'audiovisuel, chacune de ces situations de gestion est marquée par un transfert de la décision d’une part, mais aussi, d'autre part, par une découplage entre le marché reliant l'offreur au prescripteur et celui reliant le prescripteur au consommateur, ces deux marchés faisant l'objet de transactions et de dynamiques distinctes.

Par ses évolutions, le secteur de l'audiovisuel s'affirme de plus en plus comme une économie de l'intermédiation et de la prescription. L'article qui suit se propose d'en établir des lignes de caractérisation. La première partie sera consacrée à une analyse de la littérature autour de ces notions. Nous ferons ressortir que les travaux existants, en économie et en en gestion, jusqu'à maintenant se sont essentiellement focalisés sur l'émergence de formes d'intermédiation, sans étudier précisément leur fonctionnement, leur organisation et leur rôle dans la chaîne de valeur. De même, la prescription a été décrite comme fonction d'information, et non pas comme un maillon de la chaîne de valeur. L'économie de l'audiovisuel permet de mettre en évidence à la fois l'imbrication étroite de ces deux fonctions dans les opérateurs de télévision (ou " chaînes ») et les relations complexes qui s’instaurent entre ces prescripteurs et les consommateurs. La deuxième partie, rappel des grands traits de la structuration historique de l'économie de l'audiovisuel, aura pour but de mettre en évidence les différents modèles qui se sont instaurés. La troisième partie analysera ces modèles, sous l'angle du rôle des opérateurs et de la relation qui s'instaure entre eux et les consommateurs. Elle permettra ainsi de réinterpréter l'histoire récente de l'audiovisuel non plus sous un angle «business models », mais sous un angle "prescription »: l'évolution des modèles de l'audiovisuel traduit une évolution des formes de prescription sous-jacentes. 


\section{Prescription et intermédiation : une revue de littérature}

La télévision est née dans l'entre-deux-guerres. Sa diffusion dans la plupart des pays s’est étalée de la fin des années 30 (Etats-Unis) aux années 70 (Grèce par exemple). Elle partage partout dans le monde des traits communs définis dans un modèle dominant conçu aux Etats-Unis ${ }^{1}$, à la fois techniquement (TV, câble, satellite), dans ses modes de régulation (de l'audience, des fréquences et des réseaux), dans ses contenus (séries, jeux, émissions de plateaux, journal TV), dans ses modèles de chaînes (CNN, MTV...), dans ses modes de consommation.

En Europe, a prévalu pendant longtemps un modèle original de chaînes généralistes publiques (la RAI, la BBC, l'ORTF, la RTBF, l'ARD, la RTE) financées par la redevance ou l'impôt et affichant des projets culturels, sociaux et éducatifs nouveaux ${ }^{2}$. Pendant plusieurs décennies, cette «télévision de service public » a constitué un exemple et une référence quasi-exclusive, à la fois dans son modèle économique (financement par la redevance, part marginale de la publicité, monopole) et dans son modèle de programmation (chaîne généraliste et nationale, ambitions culturelles, sociales et informatives) et de diffusion (égalité d'accès des spectateurs, analogique hertzien). Il a été dominant, à quelques exceptions près, dans tous les pays d'Europe, jusqu'aux années 80.

Le développement des chaînes commerciales, apparues avec le mouvement de dérégulation des services publics, a bouleversé les caractéristiques des paysages audiovisuels nationaux. Cette évolution a porté d'abord sur les mécanismes de financement, la programmation et le schéma général des chaînes restant inchangés à tel point que les chaînes commerciales privées - construites sur ce même modèle - sont vite entrées en concurrence frontale avec les chaînes publiques (à l'image, en Italie, du groupe Mediaset de S . Berlusconi). Par la suite, le positionnement et le fonctionnement de ces chaînes ont évolué progressivement, le téléspectateur passant d'un statut d'usager à celui de consommateur, les activités de production des programmes, effectuées jusque là par les chaînes de service public, donnant lieu à un secteur de production indépendant.

\footnotetext{
${ }^{1}$ On se reportera utilement à Diberder, A. L. and N. Coste-Cerdan (1986). La télévision. Paris, La Découverte.

${ }^{2}$ Modèle qui diffère du modèle américain de référence, caractérisé notamment par la structure des networks, les relations de décrochage national / régional. on pourra se reporter à Guillou, B. and J.-G. Padioleau (1988). La régulation de la télévision. Paris, La documentation française et Simon, J.-P. (1991). L'Esprit des règles, réseaux et réglementation aux Etats-Unis. Paris, L'Harmattan.
} 
La diversité des modèles de chaîne qui se sont succédé historiquement dans l'audiovisuel et le multimédia traduit l'émergence de nouveaux paradigmes économiques dans les affaires : modèles originaux de rémunération et de contractualisation faisant intervenir de nouveaux acteurs économiques dans la chaîne de valeur ainsi que de nouveaux rapports entre opérateurs et constructeurs de matériel, fournisseurs de programmes et de contenus, diffuseurs et fournisseurs d'accès, consommateurs et utilisateurs finaux (Benghozi 2001).

La notion d'intermédiaire est devenue de plus en plus présente ces dernières années dans la littérature économique et de gestion. Si leur rôle dans les activités industrielles n’est pas nouveau, leur place a été réévaluée à la faveur du développement des technologies de l'information et de la communication, mais aussi dans le cadre d'une évolution des activités faisant une place de plus en plus grande à la composante d'information et de connaissance ${ }^{3}$.

En pratique, la généralisation du recours à la notion d'intermédiaire masque toutefois une forte ambiguïté sur la nature même de l'intermédiation engagée. À la lumière du cas de l'audiovisuel, il nous apparaît en particulier essentiel,, dans un premier temps, de distinguer les fonctions d'intermédiation et les fonctions de prescription. Dans une tradition que l'on trouve déjà exprimée par Commons (1934) et qui voit dans la transaction l'unité élémentaire de l'analyse économique, la notion d'intermédiaire, très présente chez les économistes, suppose une parité entre l'offre et la demande, elle s'appuie sur le constat que l'autonomisation d'une des fonctions de la transaction facilite l'équilibrage du marché et le développement des transactions en améliorant leur efficacité. Les économistes s'efforcent en effet de rendre compte de l'échange sans s'intéresser spécifiquement à la nature des biens et services échangés autrement que par la façon dont ils pèsent sur les comportements des agents (biens publics e.g.). Dans cette perspective, les intermédiaires sont essentiellement envisagés sous l'angle des fonctions transactionnelles qu'ils peuvent prendre en charge (financière, informationnelle, assurantielle).

La plupart des auteurs insistent sur la variété des situations d'intermédiation que l'on peut relever (par exemple Tapscott, 1999). Spulber (1996) note ainsi que les intermédiaires assurent la liquidité des marchés grâce à leur activité de référencement ainsi que par la capacité à assurer des liquidités. Brynjolfsson et al. (1999) soulignent la capacité des nouveaux intermédiaires («électroniques ») à réduire les frictions sur les marchés, en court-circuitant notamment les intermédiaires commerciaux traditionnels.

3 Pour une synthèse on pourra se reporter à Brousseau, E. (1999). The Governance of Transaction by Commercial Intermediaries: An Analysis of the Re-engineering of Intermediation by Electronic Commerce. Third Conference of the International Society for New Institutional Economics, Washington DC, USA. 
Le rapport de prescription relève aussi de figures distinctes et variées. Hatchuel (1996) distingue, pour sa part, la responsabilité opérationnelle, la responsabilité fonctionnelle, les maîtrises d'ouvrage et d'œuvre, l'animation, l'assistance, la facilitation, l'accompagnement, la consultation et la préconisation.

Comme le note Gensollen (1999), à la suite d'autres travaux menés sur l'internet (cf. Kollock, 1999) ou Brousseau, 1999), dans le schéma traditionnel de l'intermédiation, l'internet innove en permettant aux consommateurs de comparer directement les produits, sans passer systématiquement par des experts supposés compétents; dans ces cas, l'intermédiaire n'assure plus simplement une fonction d'expertise mais plutôt un rôle de régulateur. Gensollen (1999) distingue alors plus précisément plusieurs types d'acteurs concourant à l'intermédiation: les attracteurs, les agrégateurs, les convertisseurs (d'audience en clientèle) et les prescripteurs ${ }^{4}$.

Même quand on caractérise les principaux rôles des intermédiaires en envisageant d'autres composantes que la seule variable informationnelle, ces composantes concernent toujours, malgré tout, la fonction d'ajustement (référencement, logistique, sécurisation) faisant des intermédiaires des partenaires d'un échange dont la structure reste inchangée et qui leur est extérieur : les intermédiaires assurent une fonction de réarrangement du système de production et de commercialisation quand les offres des producteurs et les demandes des consommateurs ne s'ajustent pas spontanément (Lewis, 1995).

Or, l'émergence de tiers, à côté du producteur et du consommateur, ne résulte pas simplement des imperfections du marché ou des limites de la relation de transaction : dans de nombreux cas, elle tient très directement à la nature du bien ou à la structure de sa production. Pour prendre un cas très simple, les enseignants sont, structurellement, en position de recommander les ouvrages scolaires utilisés en support de cours: dans ce cas, leur fonction n'est pas de simple information ou influence mais véritablement d'injonction. Parler de prescription plutôt que d'intermédiation permet de ce fait de s’intéresser plus spécifiquement aux modalités de décision des consommateurs et aux modes de structuration des relations à la base de la prescription.

Dès lors, la question n'est plus simplement de s'interroger sur la nature des intermédiaires, les conditions de leur émergence et de leur substitution éventuelle à des formes plus traditionnelles

\footnotetext{
${ }^{4}$ Mais pour ce dernier auteur, cette dernière catégorie - les prescripteurs - n'existe pas encore car elle constitue une phase ultime, non encore réalisée, de l'intermédiation : la transmission d'information des clientèles homogènes aux entreprises productrices de façon à ce que les caractéristiques des nouveaux produits et services correspondent aux goûts forgés en commun par les consommateurs.
} 
d'échange, mais plutôt de comprendre la forme des marchés de prescription : leur modèle d'affaires et leur structure.

Paradoxalement, en gestion, même si le terme est très directement utilisé par les théoriciens de marketing, peu de travaux ont spécifiquement porté sur l'organisation de la prescription ${ }^{5}$. Les auteurs et praticiens du marketing utilisent le terme essentiellement pour distinguer les composantes des différents rôles jouées par les décisionnaires (acheteur, utilisateur, conseiller, décideur, préconisateur). Le prescripteur y est en général caractérisé, parmi d’autres, comme celui qui peut influencer de façon impérative la définition de l'achat (cf. Michel et al., 1996 ; Lendrevie et al., 2000) ${ }^{6}$. A ce titre, la plupart des travaux de marketing envisagent le phénomène de la prescription comme une variable conduisant à moduler éventuellement les stratégies d'entreprise (ciblage du marché ou communication) mais rarement comme un phénomène structurel à étudier en soi.

L'auteur ayant le plus directement identifié le phénomène et abordé la question est sans doute Hatchuel (1995). Même s'il distingue dans la relation de prescription deux dimensions, « organique » et « savante », son approche reste toutefois essentiellement attachée à la composante informationnelle. Son rôle permet à l'acheteur de s'orienter vers un offreur plutôt que vers un autre, de structurer sa représentation des biens à rechercher, de définir la valeur à attribuer à un bien : le consommateur se disqualifie lui-même comme acteur de ses propres choix en faisant appel à un tiers "prescripteur".

Pour Hatchuel, l'effet de la prescription sur le marché reste cependant indirect: il résulte d'une nécessité d'information et modifie, en retour, le champ des échanges, en rendant par exemple substituables des biens qui n'étaient pas perçus comme tels ou en participant à l'invention de nouveaux marchés. De façon plus générale, cette fonction d'invention des marchés est également notée par les auteurs ayant travaillé sur les TIC. Ils ont montré que dans le cas de produits et services immatériels ou à base d'information, les circuits commerciaux et de diffusion peuvent être intermédiés (Gille et al., 1994), en remettant en question la localisation et l'intégration des fonctions de transaction, et peuvent changer de nature en se dématérialisant (Benghozi et al., 2000). Zwass (1998) note de son côté qu'en créant ou en enrichissant des biens et services à base d'information, de nouveaux intermédiaires peuvent émerger sur de nouveaux marchés et contribuer à les redéfinir.

Ce rôle particulier des prescripteurs n'est toutefois pas analysé, chez Hatchuel, comme une composante structurelle du marché : il parle de marchés à prescripteurs et non de marché de la

\footnotetext{
${ }^{5} \mathrm{Au}$ sens de prescription sur un marché. Dans un cadre organisationnel, en revanche, la prescription signifie définir les modalités d'une tâche à accomplir ; sur ce sujet, par contre, on peut noter l'existence de nombreux travaux de GRH ou de sociologie du travail.

${ }^{6} \mathrm{Ce}$ qui, dans un contexte non-industriel, peut concerner notamment les enfants, prescripteurs d'achat dans le cadre familial.
} 
prescription. Même si cet auteur insiste sur l'existence d'une relation organique entre acheteurs et prescripteurs, il l'oppose à la relation qui existe entre offreurs et prescripteurs et qu'il limite au registre de l'information et du choix (tentative d'influence du prescripteur par l'offreur), dans une concurrence s'exerçant sans marché. C'est donc, au fond, une approche très voisine de celle que défendent les auteurs qui ont travaillé sur l'intermédiation électronique, caractérisée, pour reprendre l'expression de Gille et Mahonet (1994), par la capacité de tirer parti de la mise en correspondance de flux transactionnels et de gisements informationnels. Il est d'ailleurs intéressant de souligner que, pour Hatchuel, une des conséquences de la perception d'ordre purement cognitif de l'activité de prescription est le caractère nécessairement précaire du recours au prescripteur puisque chaque intervention assure un transfert de connaissance vers le consommateur final. Ce faisant, c'est donc bien le caractère organique et structurel de certaines relations de prescription qui est, en pratique, évacué dans l'analyse.

Dans cette perspective, il n'est pas étonnant que peu d'auteurs se soient saisis de la notion de relation d'agence (cf. Jensen et Macking, 1976) pour rendre compte du phénomène de la prescription ou de l'intermédiation autrement que de manière métaphorique. Elle fournit pourtant un cadre d'analyse permettant de rendre compte de la position du prescripteur : contrat selon les termes duquel une ou plusieurs personnes (le principal) engagent un agent pour accomplir un service pour leur compte, ce qui nécessite la délégation d'une partie du processus de prise de décision. Les travaux conduits dans cette perspective se concentrent plutôt sur la structure de propriété et de contrôle des entreprises : existence des managers, créanciers, actionnaires, moyens de maximiser leurs objectifs...

La façon de caractériser intermédiaires ou prescripteurs n'est pas seulement une question théorique, elle permet également de comprendre les modes de rémunération et les stratégies en présence. Pour les économistes, par exemple, si les acteurs assurent une fonction d’achat-revente, leur rémunération résulte de la plus-value ou du surplus résiduel des opérations. En revanche, s’ils se contentent de faciliter ou de conseiller l'échange, leur rémunération s'appuiera plutôt sur la commission que seront prêts à payer l’offreur ou le demandeur (Hackett, 1992). Buzzel (1985) montre également comment le développement des TIC conduit à renforcer les intermédiaires et les grossistes au détriment des distributeurs et producteurs. Ce qu'il voit en jeu dans ce basculement concerne notamment la capacité d'articuler des groupages et commandes importantes, la logistique, l'optimisation des encours pour les flux financiers. Pour Spulber (1996), les intermédiaires assurent d'ailleurs la contrepartie réelle des transactions entre agents économiques car ils disposent temporairement des droits de propriété qui sont transférés entre les opérateurs de la transaction, et ce sont eux, de ce fait qui assurent les risques.

Les intermédiaires ne se contentent donc pas de jouer sur les effets de volume ou d'amélioration de la transaction (Bailey, 1998). Or malgré ces travaux, l’intermédiation commerciale n’a pas intéressé en 
tant que telle les économistes. Pourtant les travaux cités montrent que la place d'intermédiaires dans les filières tient souvent à la valeur ajoutée (et non pas simplement à l'information) qu'ils apportent au produit ou service et que leur rôle est effectif dans la structuration de l'offre (et non pas simplement dans son accompagnement).

Betancourt et al. (1993) considèrent par exemple le bundling de services comme une dimension essentielle des intermédiaires commerciaux ; en effet, quand l'information sur les produits ou services est complexe ou peu transparente, les subventions croisées entre marchés permettent aux intermédiaires commerciaux de réduire les coûts et d'augmenter les opportunités de transactions (Myerson and Satterthwaite 1983). Ce point est important dans le passage de la notion d'intermédiaire à celle de prescripteur. Pour proposer un groupage des biens et services et réduire les coûts d'accès, les intermédiaires ne peuvent plus se contenter d'être de simples courtiers, ils sont de véritables acteurs de la chaîne de diffusion, ce qui peut les conduire à prendre ou détenir des droits de propriété sur les biens échangés.

Le marketing industriel a relevé depuis longtemps que les distributeurs peuvent trouver avantageux de traiter des produits en exclusivité avec un seul producteur et le degré de sélectivité de la distribution peut être analysé comme un élément du design des canaux stratégiques d'une fournisseur (Stern and Sturdivant 1987). Le bénéfice de cette spécialisation peut toutefois paraître incertain (Scherer and Ross 1990). A l'inverse, pour les fournisseurs, réduire la disponibilité des produits dans un marché très compétitif peut être contre-productif car la disponibilité, plutôt que l'action du distributeur, est le facteur principal guidant les ventes sur ces marchés (Farris, Oliver et al. 1989). Comme on le verra plus loin, ce rapport d'exclusivité des prescripteurs avec les offreurs de produits et services d'une part, des consommateurs avec les prescripteurs d'autre part, constitue un point central dans la manière dont s'organise la filière de l'audiovisuel.

Il convient donc bien d'envisager les prescripteurs en tant que «tierce partie » plutôt qu'intermédiaires. Ils interviennent à côté des producteurs et des consommateurs ${ }^{7}$ pour assurer la mise en forme de l'offre ou prendre en charge une partie de la décision de consommation (contenu ou moment où s'effectue la consommation). L'exemple des opérateurs de télévision nous permettra de l'illustrer. Avant cela, il nous faut présenter le processus de structuration historique de l'audiovisuel, pour notamment mettre en évidence l'évolution du rôle et de la place de ces opérateurs dans la chaîne de valeur.

\footnotetext{
${ }^{7}$ Hagel et Armstrong (1997) reconnaissent d'ailleurs que les nouveaux intermédiaires ont intérêt à faire évoluer leur position pour se poser en mandataire des consommateurs : le surcroît d'information qu'ils fournissent permet à ces derniers de récupérer du pouvoir de marché.
} 


\section{Le secteur de l'audiovisuel : histoire, formes et évolution}

Nous avons rappelé, au début de ce texte, que le développement des chaînes commerciales privées en Europe, avait constitué la première étape de la mutation de l'audiovisuel européen et des industries de programmes. S'ensuivit une deuxième étape d'ordre technique, avec d'abord l'émergence des chaînes à péage et des bouquets satellitaires, puis l'arrivée du numérique. Chacun de ces deux stades a donné lieu à une multiplication sensible du nombre de chaînes (apparition de chaînes privées généralistes d'abord, multiplication des chaînes thématiques ensuite) et à l'évolution de leur programmation (de programmes généralistes fédérateurs d'audience à thématiques segmentant les publics). Cet élargissement de l'offre pèsera lourdement sur la situation concurrentielle, ainsi que sur la nature des équilibres et des pratiques dans tout le secteur.

Ces évolutions se sont accompagnées de transformations plus radicales : l'arrivée de nouveaux acteurs économiques dans le champ télévisuel (les industriels de la technologie, les opérateurs de télécommunication et les opérateurs de bouquet), une approche différente du public, désormais segmenté par centres d'intérêt ou par caractéristiques sociales (dans les chaînes thématiques mais également dans les chaînes généralistes), l'apparition de modes élargis de rémunération et de modèles économiques nouveaux puisque l'identification d’un public ciblé permet de lui proposer des programmes adaptés pour lesquels il est disposé à payer spécifiquement (sous forme d'abonnement, ou de pay per view), en engageant donc un travail de programmation (et une production) beaucoup plus précis qu'auparavant.

La montée en puissance du secteur commercial de la télévision s'est aussi accompagnée d'une transformation radicale sur le plan industriel. À l'heure où les progrès techniques favorisent la multiplication de l'offre et accroissent la demande en termes de fourniture de contenus, cette industrie constitue un secteur d'investissement attractif. Les développements récents ont notamment été marqués par une intensification de la concentration dans les filières et une internationalisation grandissante : la constitution progressive de grands groupes privés, à l'image de Vivendi Universal, en est une des illustrations les plus flagrantes.

\section{Convergence / uniformisation entre les modèles de télévision}

Le mouvement d'homogénéisation autour d'une organisation économique de la télévision et de pratiques communes tient à plusieurs facteurs de natures très différentes. Le premier résulte des effets de contingence technologique : le développement du câble, du satellite et du numérique hertzien ont 
permis (et conduit à) une multiplication des programmes potentiels en accélérant la remise en cause des monopoles publics. Un deuxième facteur résulte des comportements de mimétisme et d'imitation de modèles éprouvés, d’une chaîne à l'autre : Canal + s'est largement inspirée de la chaîne américaine du câble HBO à la fois dans sa programmation, sa stratégie à l'égard des fournisseurs de programmes et son positionnement commercial ; elle a ensuite dupliqué le concept dans les autres pays où elle s'est implantée (Italie, Espagne, Belgique...). Le troisième facteur, d’ordre économique, est sous-tendu par les importants effets d'échelle que permet la télévision : le coût de la diffusion restant marginal par rapport aux investissements de production, les acteurs économiques ont fortement intérêt à une large diffusion de leurs programmes (quittes à réduire les prix de vente des œuvres une fois celles-ci amorties). Cela conduit à un fort mouvement de brassage et de standardisation des programmes et des chaînes par la diffusion mondialisée des programmes (films et séries), ou la diffusion et adaptation locales de concepts mondiaux (Qui veut gagner des millions?, Loft Story, séries policières récurrentes...). La constitution progressive de groupes multimédias mondialisés (Berlusconi, Turner, Vivendi) accentue encore le phénomène.Il est important de noter que tous ces facteurs de convergence ont donc plutôt contribué à renforcer le poids des diffuseurs- distributeurs.

\section{Une évolution des modes de consommation et du rapport au spectateur}

Les mouvements stratégiques auxquels a donné lieu la filière audiovisuelle se sont doublés d'une transformation des pratiques des spectateurs dans leur rapport à la télévision, ce qui a également accentué le caractère central de la fonction de programmation-diffusion dans l'organisation de la chaîne de valeur. Depuis une vingtaine d'année, les grilles de programmation se sont allongées et il y a de plus en plus de personnes disponibles la journée et même la nuit devant la télévision. La consommation moyenne par spectateur a fortement augmenté en l'espace de vingt ans pour atteindre couramment, quel que soit le pays, de trois à quatre heures par jour en moyenne.

De plus, la logique du marché des programmes s'est rapidement imbriquée dans la logique des carrefours horaires. Ces derniers se sont précisés et affinés dans les grilles de programmation. Ils confortent la segmentation du marché des téléspectateurs et la standardisation des programmes, leur durée (26 et 52 minutes), les grilles et les créneaux de programmation : les carrefours horaires (day access - prime time ...) sont précisément définis et à chaque tranche horaire correspond désormais des types de programmes tout aussi bien définis : jeux, divertissements et sitcoms dans l'access prime time, fiction TV ou cinéma, variétés ou sports pour le prime time par exemple.

Cette évolution a conduit l'audiovisuel à glisser d'une situation où les chaînes étaient des simples supports de diffusion à une position où elles organisent et proposent une véritable structuration de la consommation. Dans ce dernier cadre, c’est alors moins le repérage d'un programme donné qui est 
important pour le consommateur que l’identité de la chaîne, garante de la qualité et de la nature de sa programmation $^{8}$.

Le changement de nature de la relation au téléspectateur / consommateur potentiel s'est accompagné, parallèlement, d'une utilisation différente des mesures d'audience. Les chaînes ont dépassé une logique de production où la sanction éventuelle par l'audience se manifestait surtout ex post (en attendant le résultat de l'audience pour savoir si on gardait l'émission à l'antenne ou non). Elles adoptent désormais de plus en plus une logique de design to cost où la part de marché espérée détermine le volume publicitaire souhaité, les recettes potentielles et le montant du budget susceptible d'être attribué pour la production de la tranche horaire considérée : il ne s'agit donc plus simplement d'espérer l'audience la plus large possible, mais de s'organiser en fonction du volume de spectateurs attendus à une heure donnée. Dans une telle perspective, l'organisation de la grille de programmation se veut le reflet d'une segmentation du marché des téléspectateurs aux diverses heures de la journée, en fonction de la consommation, individuelle ou familiale qui y correspond. La logique marketing de niche devient prédominante tant au niveau de la conception des programmes que du positionnement des chaînes (cf. chaînes thématiques).

Dès lors, le public a été progressivement - puis quasi exclusivement - perçu dans une logique de marché et d'audience où le spectateur est envisagé comme consommateur potentiel (des produits dont on fait la publicité). Les chaînes se sont de ce fait placées dans un cadre différent de celui qui prévalait initialement dans le modèle historique de la télévision européenne, c'est-à-dire où le spectateur était l'usager d'un service public, devant pouvoir bénéficier des missions explicites du service public, tel que l'accès à la culture, à l'information. Le renforcement de la compétition entre opérateurs, le développement des pratiques d'abonnement (câble, satellite, chaînes à péage) et l'émergence consécutive d'une logique de marque ont conduit les chaînes à abandonner la position de simple distributeur sélectionnant des œuvres et les mettant à disposition d'un public qui détermine ses choix en fonction de la nature de l'offre proposée. Elles se positionnent désormais dans un rôle de prescripteur à qui le consommateur fait confiance pour choisir le bon programme à sa place au moment où il aura envie de voir la télévision : dans un tel cadre, la décision prépondérante n’est dès lors plus la sélection d’un programme mais le choix d’un opérateur.

Cette fonction de prescription assurée désormais par les chaînes a influencé très directement leur comportement dans les relations avec les offreurs de programmes : politiques d'acquisition des droits, exclusivité, prix des programmes. Au niveau de la production, l'évolution des pratiques commerciales

\footnotetext{
${ }^{8}$ La forme actuelle des programmes de télévision publiés dans la presse est une parfaite illustration de glissement. Alors que les chaînes généralistes (publiques, privées ou payantes) font l'objet d'un compte-rendu extensif de leurs horaires, les chaînes thématiques ne comportent que des renvois sommaires à quelques émissions (pas nécessairement les plus importantes d'ailleurs) ; il faut noter que c'est déjà la situation que connaissent les radios.
} 
a eu plus particulièrement pour effet de provoquer un élargissement des coûts de programme et de leurs prix d'achat. Plus un programme est porteur d'audience, plus sa diffusion est avantageuse pour la chaîne et plus son prix d'acquisition tend à augmenter... et inversement, et ce, qu'il s'agisse de films de cinéma ou de matches de football. Les programmes à la fois rares et potentiellement attractifs font l'objet de surenchères qui se traduisent par une inflation considérable ${ }^{9}$.

\section{Vers un nouvel équilibre}

L'histoire de l'audiovisuel a vu se construire plusieurs modèles économiques, qui coexistent aujourd'hui $^{10}$ : télévision hertzienne gratuite, télévision hertzienne commerciale, télévision à péage, bouquets câble ou satellite, paiement à la séance... Chaque situation correspond à des déploiements, des types de programmes, des types d'actionnariat, de financement et de régulation différents ${ }^{11}$.

\begin{tabular}{|l|l|l|l|}
\hline MODELE & OBJECTIF & CONTEXTE ECONOMIQUE & CONTRAINTES \\
\hline NETWORK & $\begin{array}{l}\text { Optimiser le public, la } \\
\text { couverture et les recettes } \\
\text { publicitaires }\end{array}$ & $\begin{array}{l}\text { double concurrence : au niveau } \\
\text { des réseaux et des stations } \\
\text { locales }\end{array}$ & $\begin{array}{l}\text { Position d'intermédiaire entre } \\
\text { niveau national et niveau local }\end{array}$ \\
\hline AUDIOVISUEL & $\begin{array}{l}\text { assurer des fonctions de } \\
\text { service public (éducation, } \\
\text { culture, politique...) }\end{array}$ & $\begin{array}{l}\text { financement public et contrôle par } \\
\text { tutelle ou instance de régulation }\end{array}$ & $\begin{array}{l}\text { limitation des financements et } \\
\text { du nombre des chaînes, } \\
\text { gouvernance publique }\end{array}$ \\
\hline $\begin{array}{l}\text { CHAINE } \\
\text { COMMERCIALE } \\
\text { GENERALISTE }\end{array}$ & $\begin{array}{l}\text { Développer une audience de } \\
\text { masse }\end{array}$ & $\begin{array}{l}\text { financement par la publicité et } \\
\text { valorisation de l'effet de marque }\end{array}$ & $\begin{array}{l}\text { modèle dérivé des chaînes } \\
\text { publiques, optimisation de la } \\
\text { couverture de diffusion }\end{array}$ \\
\hline $\begin{array}{l}\text { CHAINE } \\
\text { THEMATIQUE }\end{array}$ & $\begin{array}{l}\text { Spécialisation sur un marché } \\
\text { de niche }\end{array}$ & $\begin{array}{l}\text { intégration dans un bouquet de } \\
\text { programmes, envergure } \\
\text { modulable }\end{array}$ & $\begin{array}{l}\text { coupure entre chaîne et } \\
\text { bouquet, non maîtrise des } \\
\text { supports de diffusion }\end{array}$ \\
\hline
\end{tabular}

Sous l'effet de ces évolutions, les modèles historiques de l'audiovisuel sont soumis à de nombreuses pressions : remise en cause de la légitimité du financement public, implosion sous l'effet de la concentration et de la croissance progressive conduisant à réduire progressivement la place des opérateurs et producteurs indépendants ${ }^{12}$, concurrence nouvelle des chaînes thématiques numériques

\footnotetext{
${ }_{9}^{9} \mathrm{~A}$ contrario, les programmes à plus faibles audiences sont acquis à des prix inférieurs à leur coût de fabrication.

${ }^{10}$ On retrouve des configurations semblables dans des secteurs très éloignés (grande distribution et VPC par exemple) : Cf. P-J. Benghozi, "Relations interentreprises et nouveaux modèles d'affaires, Revue Economique, vol. 52, N hors série "Economie de l'Internet », octobre 2001.

${ }^{11}$ Cf. Le Diberder et alii, op. cit.

12 Une autre limite du modèle actuel réside dans la nature effective de l'indépendance dont se prévalent nombre de producteurs, artistes, médias et journalistes mais qui sont, de fait, directement sous l'influence des grands groupes (tels que Canal +, Universal...) (par le biais de l'actionnariat, de contrats de production ou de first option, du poids des contrats publicitaires). De ce point de vue, le problème des échanges internationaux ne sont pas plus importants à prendre en compte que ceux qui se posent au niveau national; ces derniers tiennent aux conflits entre groupes intégrés et
} 
(câble et satellite) rognant sur leurs ressources et appelant des stratégies plus globales et plurisectorielles sur lesquelles elles se heurtent à des opérateurs de plus grande taille qu'elles, tension sur le marché de la production et de la fourniture de droits résultant d'un accroissement très fort de la fourchette de prix entre programmes connaissant une inflation des coûts et programmes sous-payés.

Ces tensions propres au marché de l'audiovisuel sont accentuées par le développement de l'internet et des supports multimédias qui conduit à une profonde redistribution des cartes, aussi bien d'un point de vue économique qu'en matière de pratiques des consommateurs et de types de programmes produits. L’arrivée de l'internet et du numérique dans la télévision grand public, avec comme conséquence la multiplication des chaînes d'une part, des terminaux et des modes de réception d'autre part, soulève la question d'une nouvelle transformation structurelle de ce secteur, de ses acteurs, des modalités de programmation et des pratiques de consommation des programmes. Les modèles organisationnels et économiques qui se développent à partir de l'audiovisuel autour du multimédia ne sont pas une simple adaptation de configurations élaborées dans la télévision. L’apparition de formes radicalement nouvelles de marché (portails d'information, services à valeur ajoutée) et le poids grandissant des fournisseurs de technologie a généré l'apparition de modèles originaux de rémunération et de contractualisation voyant l'apparition de nouveaux intervenants dans les filières du multimédia ainsi que de nouveaux rapports entre opérateurs et constructeurs de matériel, fournisseurs de programmes et de contenus, diffuseur et fournisseurs d'accès, consommateurs et utilisateurs finaux (cf. Benghozi, Paris, 2001).

Il s'établit un équilibre entre les différents supports de l'audiovisuel : câble, satellite, payant, hertzien, réseaux numériques... Plutôt que des marchés en concurrence, il faut de plus en plus y voir des marchés en complémentarité veillant à positionner chacun des supports de façon spécifique en évitant leur cannibalisation réciproque ${ }^{13}$.

\section{Une évolution du métier des chaînes}

En termes de service offert, ces modèles voient s'établir des relations différentes entre chaînes et consommateurs. Cette différence s'exprime plus particulièrement dans les modes de tarification et de financement des chaînes par les consommateurs.

indépendants, à la généralisation de pratiques de production "à l'hollywoodienne" privilégiant des créneaux et des thématiques spécifiques (marchés des adolescents...), aux modes de valorisation et de commercialisation favorisant la starisation et la massification de la consommation.

${ }^{13}$ cf; les débats sur la "troisième fenêtre" visant à prévoir la commercialisation sur chaîne satellite en organisant de façon concertée et organisée la temporalité de la commercialisation des films de cinéma sur différents supports : salles, vidéo après 9 mois, chaîne payante après un an, chaîne généraliste après deux ans... 


\section{Le modèle de la télévision « gratuite »}

Le modèle de la télévision "gratuite » est celui des chaînes hertziennes traditionnelles : elle n’est gratuite qu'au sens où le consommateur ne paye pas directement la chaîne. Dans ce modèle, le consommateur a le choix entre un nombre limité de chaînes (programmateurs) et donc, de programmes. La rémunération de la transaction se fait selon deux modèles principaux : le modèle de la publicité (chaîne commerciale généraliste), dans lequel des annonceurs payent pour pouvoir faire passer des messages aux consommateurs, et le modèle de la redevance (audiovisuel public) dans lequel la programmation est financée par une taxe redistribuée aux chaînes.

\section{Le modèle du pay-per-view}

Dans ce modèle, le consommateur achète le droit d'accéder au programme diffusé à l'instant $t$ (éventuellement, en multi-diffusion). D’une certaine façon, la logique pour le diffuseur reste celle d'une agrégation de masse, similaire à celle de la télévision traditionnelle, la différence tenant au modèle économique, basé dans ce cas-ci non pas sur la publicité mais sur le consommateur.

\section{Le modèle de la télévision à péage}

Dans ce modèle, le consommateur, en amont de son choix de programmes, peut décider d'élargir le nombre de chaînes dont il souhaite disposer en souscrivant un abonnement à une chaîne à péage. La rémunération des consommations est un abonnement forfaitaire qui donne accès, sur la durée de l'abonnement, à l'ensemble des programmes.

Contrairement au modèle précédent, ce modèle suppose que le consommateur ait une visibilité sur les programmes qui seront proposés, ou une confiance dans le programmateur : il accepte de payer pour avoir une chaîne supplémentaire si cette chaîne lui offre des programmes qu'il ne trouve pas ailleurs (exclusivité), si elle lui garantit une qualité éditoriale (choix des programmes) ou une qualité de service (multi-diffusions, cryptage de certains programmes...).

\section{Le modèle des bouquets (câble ou satellite)}

Dans ce modèle, le consommateur s'abonne pour élargir le nombre de chaînes dont il dispose. L'abonnement à un opérateur câble ou satellite lui donne accès à plusieurs nouvelles chaînes, distinctes du diffuseur. En outre, l'achat d'une parabole donne accès gratuitement à un certain nombre de chaînes, relevant de la télévision gratuite (chaînes hertziennes généralistes nationales ou étrangères). L'abonnement consiste essentiellement à acheter un catalogue de droits et une capacité de négociation (capacité du diffuseur à obtenir telle ou telle exclusivité). 


\section{Le modèle de la video on demand}

Ce modèle s'est développé aux Etats-Unis mais n’a pas encore trouvé de forme viable en Europe. À la frontière entre la télévision et l'internet, il consiste en ce que le consommateur accède à une base de programmes (non situés dans le temps) parmi lesquels il fait un choix. Plusieurs modèles économiques sont possibles : abonnement ou paiement à la séance.

\begin{tabular}{|l|c|c|c|}
\hline \multicolumn{1}{|c|}{$\begin{array}{c}\text { Décision / abonnement } \\
\text { consommation }\end{array}$} & Multichaînes & A la chaîne & Au programme \\
\hline Temporel & Canalsat / TPS & Canal + & Pay per View \\
\hline Détemporalisé & \multicolumn{2}{|c|}{ Net médias } & Video on Demand \\
\hline
\end{tabular}

Les caractéristiques économiques et institutionnelles de l'industrie audiovisuelle contribuent ainsi, d'un côté, à façonner et sélectionner certains modes d'organisation plus particulièrement efficaces et adaptés à une valorisation économique. Ces organisations se constituent, d'un autre côté, comme modèles de référence et génèrent ensuite des phénomènes d'imitation : ces schémas de fonctionnement constituent des cadres communs à partir desquels chaque pays, chaque chaîne adoptera des modalités particulières, soit du fait des caractéristiques locales de son marché, soit par volonté de se différencier des chaînes concurrentes, soit pour mieux tenir compte de son expérience et s’adapter progressivement à un contexte économique très évolutif. Dans le cadre d'un schéma de développement analogue, chaque pays a ainsi pu développer et construire une spécificité lui permettant de protéger et de soutenir ses industries nationales, qu'il s'agisse de celle des programmes ou des équipements. La diversité est portée à la fois par des caractéristiques techniques (modalités de diffusion et de transport du signal hertzien, câble, satellite), les normes de codage (Pal, Secam, NTSC, numérique) et les structures des réseaux (network, audiovisuel public, commercial, spécialisé).

La lecture des modèles, nouveaux ou anciens, existants ou en développement, sous l'angle des relations qui s'établissent entre la chaîne et le consommateur permettent de mettre en évidence une diversité du métier d’opérateur de télévision. Derrière les modèles économiques, il y a donc aussi des modèles de service, que nous nous efforçons de caractériser et de modéliser dans la partie suivante.

\section{Modélisation}

Dans cette partie, nous questionnons les relations qui s'établissent entre l'offre de programmes, les consommateurs et les diffuseurs, en fonction notamment des différents modèles de l'audiovisuel que 
nous venons de mettre en évidence. Il s’agit de réinterpréter le processus historique que nous venons d'esquisser, sous l'angle de l'évolution de la fonction des chaînes. L'hypothèse sous-jacente à notre démarche est que les évolutions technologiques ont permis au service proposé par les chaînes - la prescription - de s'enrichir progressivement, et de passer d'un modèle de distribution que nous qualifierons de prescription unique à un modèle de prescription multiple.

\section{L’économie de l'audiovisuel : une caractérisation}

Dans une économie classique, le consommateur choisit un bien parmi l'offre existante. Dans l'économie de l'audiovisuel, le consommateur est tenu de recourir à un vecteur technique (le terminal de télévision) et de passer par un intermédiaire technique (le diffuseur) pour faire acte de consommation (voir une émission). L'acte de consommation d'un programme passe par un opérateur de diffusion, le diffuseur, qui assume aussi le rôle du distributeur ou d'une institution de marché, en tant que lieu de rencontre de l'offre et de la demande ${ }^{14}$.

Traditionnellement, l'audiovisuel représentait l'ensemble des produits qui transitaient par la place de marché «téléviseur ». La numérisation et les NTIC ont provoqué la mise en place de ponts entre différents terminaux (téléviseur, ordinateur, téléphone...) due à l’homogénéisation technique des différents produits auxquels ces différents terminaux permettaient d'accéder (voix, fichiers informatiques, produits audiovisuels...). On appellera économie de l'audiovisuel l'économie constituée par les transactions de produits audiovisuels, indépendamment des terminaux (téléviseurs, ordinateurs, téléphones mobiles, assistants personnels...) et des modes de diffusion (voie hertzienne, internet, câble...) utilisés. Par produits audiovisuels, ou programmes, nous entendons les produits constitués d'images, animées ou non, et de son, et conçus de manière industrielle pour la diffusion à grande échelle ${ }^{15}$. Nous appellerons diffuseur un opérateur qui diffuse des programmes.

\section{Analyse du processus de la demande dans l'audiovisuel}

La rencontre de l'offre et de la demande, dans l'audiovisuel, se fait via la place de marché que constitue le terminal, et qui est alimentée par les diffuseurs. Le consommateur doit choisir dans l'offre existante à l'instant t.

Nous distinguons deux paradigmes. Dans le paradigme « push ", l’offre est située dans le temps ; dans le paradigme «pull», le consommateur peut avoir accès à l'ensemble de l'offre indépendamment du temps. Cette différenciation correspond à la différence entre la télévision historique, où l'on ne peut choisir que parmi les programmes diffusés à l'instant $t$, et l'internet, où l'on

\footnotetext{
${ }^{14}$ Marché est ici utilisé au sens concret, celui de lieu (de rencontre de l'offre et de la demande).

${ }^{15}$ Dans l'audiovisuel, on distingue les programmes de flux des programmes de stocks : les premiers sont réputés perdre leur valeur marchande une fois diffusés (journaux télévisés, événements sportifs, jeux...), tandis que les seconds la conservent (films, magasines...). Dans cet article, nous ne faisons pas la distinction et considérons que tous les programmes relèvent d'une même offre.
} 
va puiser parmi l'ensemble des programmes mis à disposition. Les diffuseurs en paradigme " push » seront appelés programmateurs : ils font un choix de programmes et les agencent dans le temps ${ }^{16}$.

Dans le paradigme «push », le choix du consommateur porte à la fois, de façon indissociable, sur un programme et sur un programmateur. À l'instant t, le programme se confond avec le programmateur : faire le choix d'une chaîne (d'un programmateur) à l'instant $t$, c'est faire le choix du programme proposé par cette chaîne.

\section{Le processus de la demande dans le paradigme " push »}

$$
\begin{gathered}
\text { Choix d'un programme = } \\
\text { entrée sur le marché (mise en marche du terminal) + } \\
\text { choix d'un programmateur-programme (choix d'une chaîne) }
\end{gathered}
$$

La décision préalable à l'acte de consommation se présente donc comme un choix à trois dimensions : l'instant $\mathrm{t}$ de consommation, le programme et le programmateur.

$$
\mathrm{D}=(\mathrm{t}, \mathrm{P}, \mathrm{p})
$$

où $\mathrm{D}$ est le choix effectué par le consommateur, $\mathrm{P}$ le programmateur et $\mathrm{p}$ le programme

Ces trois variables sont liées, dans la mesure où le choix de t et de $\mathrm{P}$ définit $\mathrm{p}: \mathrm{p}=\mathrm{p}(\mathrm{t}, \mathrm{P})^{17}$.

Plus précisément, on peut envisager plusieurs formes de décisions de consommation :

- le consommateur consulte l'offre hebdomadaire (le guide des programmes) et décide de consommer un programme précis : son choix porte sur p, et t et P sont imposés ;

- à un instant donné, le consommateur fait un choix parmi les programmes diffusés : son choix porte sur p, t étant fixé, et P est imposé ;

- à un instant donné, le consommateur décide de faire confiance à un programmateur : son choix porte sur P, t étant fixé, p est imposé.

Cette analyse de la « demande » met en évidence plusieurs conclusions :

- le processus de choix du consommateur ne relève pas du choix de l'économie classique ; il ne consiste pas toujours en une optimisation sur l'ensemble de programmes existants, en fonction de préférences ; il porte sur une offre médiatisée par des programmateurs, ou sur les programmateurs eux-mêmes ;

\footnotetext{
16 La meilleure traduction française pour rendre compte de ces deux modèles serait «paradigme flux » et «paradigme stock ». Seulement, cette terminologie existe déjà dans l'audiovisuel, avec un sens un peu différent, comme nous le verrons.

${ }_{17}$ Pour plus de précision, il faudrait considérer que la fonction de programmation $\mathrm{p}(\mathrm{t})$ n'est pas continue mais discrète : le choix des programmateurs consiste d'une part en un découpage du temps en créneaux et d'autre part en un remplissage des créneaux par des programmes de l'offre. Par souci de simplification de la formalisation, nous présentons la fonction de programmation comme une fonction continue.
} 
- le rôle des programmateurs est multiple et selon les formes de consommation, l'un ou l'autre de ces rôles est mis en avant : intermédiaire technique ou prescripteur.

\section{La rencontre offre-demande dans le paradigme " push "}

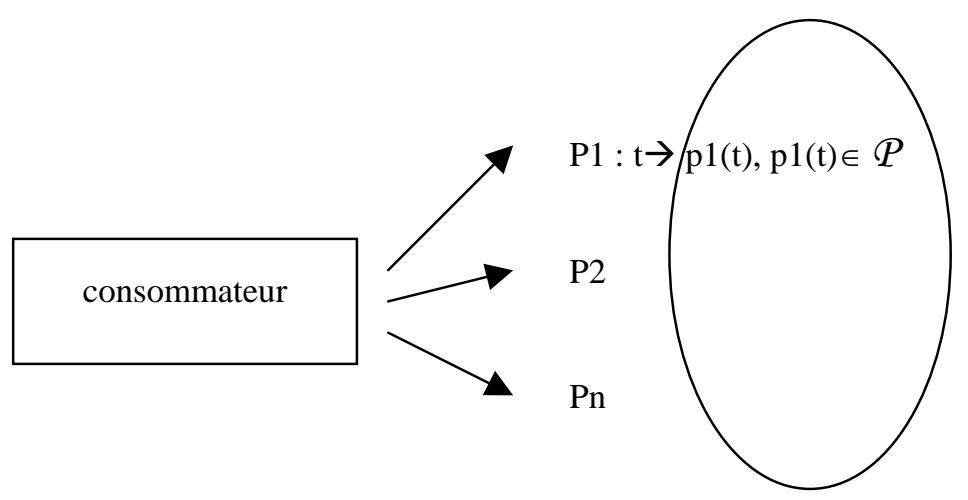

PROGRAMMATEURS OFFRE $\mathcal{P}$

Dans le paradigme « pull », la dimension temporelle n'intervient plus. Le choix porte sur le diffuseur, puis sur l'un des programmes proposés par le diffuseur.

Le processus de la demande dans le paradigme « pull »

Choix d'un programme $=$ entrée sur le marché (mise en marche du terminal)

+ choix d'un diffuseur

+ choix d'un programme

Dans ce cas, les dimensions $\mathrm{P}$ (le diffuseur) et p (le programme) deviennent indépendantes du temps ${ }^{18}$.

\section{Modèles de l'audiovisuel et formes de prescription}

L'intermédiation entre le consommateur et l'offre implique donc différentes fonctions d'ordre à la fois informationnel et organique : l'opérateur de télévision sélectionne des programmes, les programmes et les diffuse.

Le service audiovisuel (le processus d'offre dans l'économie de l'audiovisuel)

\footnotetext{
${ }^{18} \mathrm{~A}$ quelques nuances près : I'offre des diffuseurs évolue toujours, mais le choix dans un catalogue donné n'implique plus le
} choix d'un créneau horaire. 


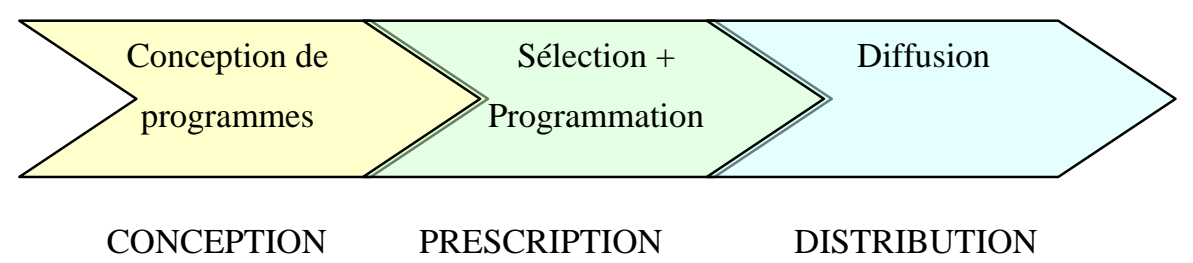

Nous nous intéressons ici uniquement à la fonction de prescription. Notamment, nous n'étudions pas les relations de la programmation avec la conception d'une part et de la programmation avec la diffusion d'autre part ${ }^{19}$. C'est cette prescription qui apparaît, du point de vue du client, comme l’intermédiaire entre le marché et lui ; c'est autour de la prescription que se gère la relation aux clients.

L’intermédiation, et la prescription, prennent des formes différentes selon le paradigme considéré. Dans le paradigme « push » - l'audiovisuel traditionnel dans lequel le consommateur a le choix entre plusieurs chaînes qui diffusent chacune un flux temporel de programmes -, l'intermédiaire technique, la chaîne, est aussi programmateur.

Plus précisément, le paramètre déterminant est le « degré de multiplicité de l’offre » : il traduit le fait que l'offre proposée par le diffuseur à l'instant t est constituée d'un programme unique (le paramètre prend la valeur 1), de programmes multiples (n) ou d'une infinité de programmes $(\infty)$.

Selon ce paramètre, les modèles de l'audiovisuel se structurent en deux catégories, qui donnent lieu chacun à un rapport de prescription particulier :

a. modèle de la prescription unique

Ce cas correspond aux modèles audiovisuels dans lesquels la programmation est unique : télévision gratuite, pay-per-view, télévision à péage. Dans ces modèles, l’offre de programmes n’est pas directement accessible au consommateur : elle est filtrée par le programmateur. Du point de vue du consommateur, la chaîne masque l'offre, mais aussi s'efface devant le programme, qui fait le réel objet du choix. Elle apparaît réellement comme un intermédiaire technique, au point qu'elle est assimilable à un bouton sur le poste de télévision. Cela transparaît dans le fait que les chaînes étaient, aux temps de la télévision publique, identifiées et nommées par les consommateurs selon leur numéro (la Une, la Deux...). La « transparence » des marques traduisait bien la transparence du rôle des chaînes pour les consommateurs.

\footnotetext{
${ }^{19}$ La relation entre les concepteurs de programmes et les programmateurs est en règle générale une relation de fournisseur. Elle peut être internalisée ou externalisée, donner lieu à différents types de relations (achat ponctuel, achat d'une série de programmes, contrat de fourniture de longue durée, cession d'un créneau, achat au forfait, reversement de royalties...).
} 
Dans ce contexte, le rapport de prescription qui s'établit est ambigu : d'un côté, le consommateur reste maître de ses choix, mais ils s’opèrent parmi une offre filtrée par les chaînes ; de l'autre, le programme est imposé dès lors que le programmateur est choisi.

Modèle de la prescription unique

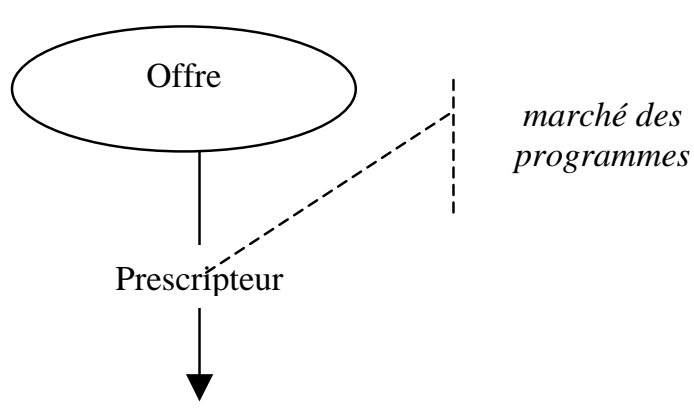

Consommateur

Avec la possibilité du cryptage, s’est développée la possibilité d’abonnements, ce qui a engagé une transformation du rapport au consommateur. L'un des changements induits est que l'indicateur de performance principal n'est plus l'audimat (audience, c'est-à-dire nombre de consommateurs à l'instant t), mais le nombre d'abonnés. Dans la télévision à péage, la relation d’abonnement traduit l'instauration d'une relation de prescription qui reste d'ordre unique mais est nettement plus affirmée : le consommateur confie au diffuseur le soin de choisir pour lui des programmes. En souscrivant un abonnement, il élargit son offre de programmes (variété de choix), il accède à des programmes en exclusivité, et il se donne la possibilité d'avoir accès à une offre de programmes qui lui corresponde.

b. Modèle de la prescription multiple

Ce modèle de prescription correspond aux modèles audiovisuels à programmation multiple (bouquets, video on demand...). Cette catégorie recouvre les modèles du paradigme « pull » et certains modèles du paradigme « push », en particulier les bouquets. Dans ces modèles, l'offre est de nouveau visible pour le consommateur. Le rôle du diffuseur évolue vers un modèle de prescription différent : il ne propose plus un programme unique, mais sélectionne un certain nombre de programmes qu'il met à disposition de ses clients.

Le modèle des bouquets démultiplie le processus de choix : l’opérateur choisit un certain nombre de chaînes, le consommateur s'abonne à un certain nombre d'entre elles, et fait le choix d'un programme à l'instant t. Le rapport de prescription qui s'établit est plus complexe, du fait qu'apparaissent deux niveaux d'intermédiaires - l'opérateur du bouquet, et les programmateurs des chaînes du bouquet - : ainsi l'opérateur fait-il une sélection de chaînes pour constituer son bouquet, lesquelles établissent leur programmation, le consommateur faisant son propre choix parmi les programmes du bouquet. 
Dans ce modèle de prescription, apparaissent ainsi trois marchés : le marché des programmes existe toujours entre l'offre et le consommateur, un marché de l'exclusivité s'établit entre le diffuseur et l’offre, un marché des droits d'accès entre le consommateur et les diffuseurs.

\section{Modèle de la prescription multiple}

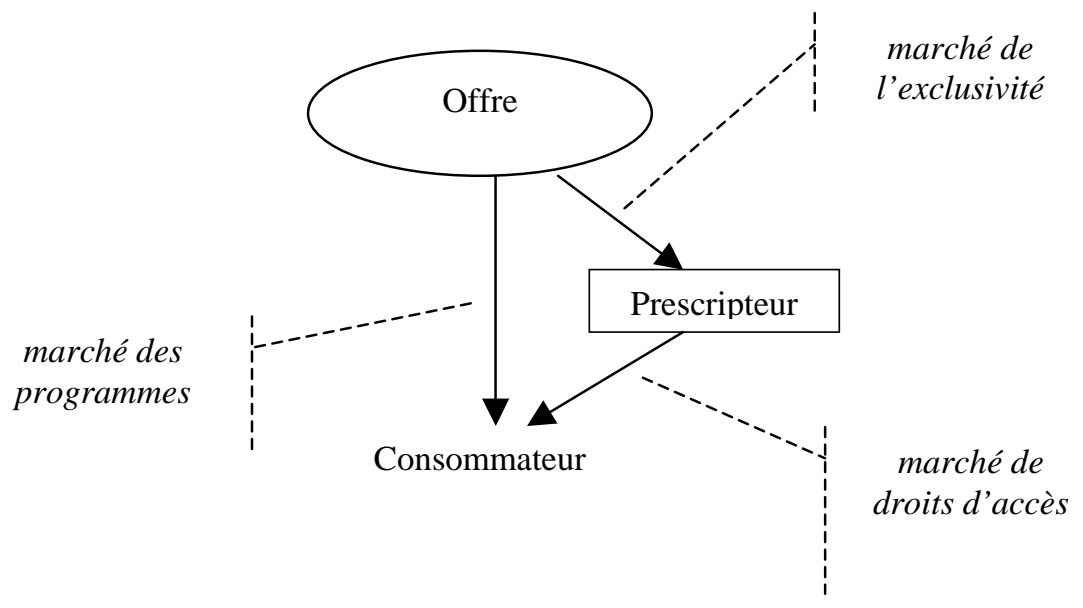

\section{Modèles de l'audiovisuel et relation commerciale au consommateur}

Le passage du modèle de prescription unique au modèle multiple fait émerger deux marchés nouveaux entre le consommateur et le prescripteur, et entre le prescripteur et l'offre, qui modifient la relation commerciale entre le consommateur et le diffuseur. Leur relation prend place sur un marché des droits d'accès : le consommateur achète à l'avance un droit d'accès à l'ensemble de l'offre proposée. Apparaît ainsi un second paramètre important pour la caractérisation des modèles de l'audiovisuel : le paramètre «durabilité de la relation entre le diffuseur et le consommateur », qui prend les valeurs « relation d'abonnement » ou « pas de relation d'abonnement ».

L'observation des modèles de l'audiovisuel, et leur caractérisation par rapport à ces paramètres, met en évidence qu'à l'exception du modèle de la télévision à péage, ils sont globalement liés : il n’y a pas de relation d'abonnement pour les modèles relevant de la prescription unique, il existe une relation d'abonnement pour les modèles relevant de la prescription multiple.

Nous pouvons résumer l'ensemble de ces modèles dans un tableau à trois dimensions.

\begin{tabular}{|c|c|c|c|}
\hline & & Abonnement & Non abonnement \\
\hline \multirow{2}{*}{ 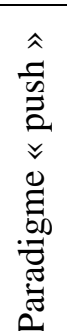 } & $\begin{array}{c}\text { Offre unique } \\
P_{i}(t)=\left\{p_{i}(t) \in P\right\}\end{array}$ & TV à péage & $\begin{array}{c}\text { TV gratuite, pay per } \\
\text { view }\end{array}$ \\
\hline & $\begin{array}{c}\text { Offre multiple } \\
P_{i}(t)=\left\{p_{i}^{j}(t), i \in\{1, \ldots, n\}\right\}\end{array}$ & Bouquet & \\
\hline
\end{tabular}




\begin{tabular}{|c|c|c|}
\hline 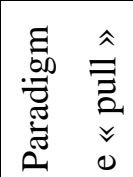 & $\begin{array}{c}\text { Offre atemporelle } \\
P_{i}(t)=P_{i}, \bigcup_{i} P_{i}=P\end{array}$ & Video on demand \\
\hline
\end{tabular}

$P_{i}$ représente à la fois l'opérateur et l'ensemble de son offre de programmes.

$P_{i}(t)$ est l'offre de l'opérateur i à l'instant $\mathrm{t}, p_{i}^{j}(t)$ le jème programme diffusé par l'opérateur $P_{i}$ à l'instant t, $P$ l'ensemble des programmes existants.

L'histoire technique et économique de l'audiovisuel et l'évolution de ses modèles économiques traduit l'imbrication de deux mouvements: structuration du marché d'un côté, évolution du rapport de prescription de l'autre. Le rôle des chaînes évolue d'une fonction d'intermédiation technique pure à une fonction qui intègre une fonction de prescription de plus en plus riche. Parallèlement, en tant qu'intermédiaire technique, et de porte d'entrée sur le marché, elles ont contribué à structurer l'offre : en la polarisant, elles s’affirment comme des « pôles de prescription ».

L'analyse sous cet angle soulève un certain nombre de questions qui nous semblent intéressantes. D’abord, à la place particulière que nous avons donnée à la télévision à péage fait singulièrement écho les difficultés que connaît aujourd'hui ce modèle dans nombre de pays européens. N'a-t-on pas en quelque sorte montré que ce modèle était désormais dominé, car les évolutions technologiques (diffusion numérique) ont permis de proposer à de nouveaux opérateurs - les bouquets - de proposer un service identique (exclusivité, qualité des programmes, qualité de service), mais enrichi de la multiplicité ?

La lecture du précédent tableau soulève une autre question importante : est-ce que l'augmentation du nombre de chaînes dans le cas d'une offre multiple fait passer à un modèle équivalent au modèle pull. En d'autres termes, quand n tend vers l'infini, le modèle de l'offre multiple tend-il vers le modèle de l'offre atemporelle ? Ou encore, à grand nombre de chaînes, y a-t-il une différence, dans le service au consommateur, entre le modèle push (la TV) et le modèle pull (l'internet) ?

\section{Conclusion}

L'analyse de la relation entre consommateurs et chaînes dans l'audiovisuel, la mise en évidence de l'ambivalence qui la caractérise - la chaîne est à la fois place de marché, intermédiaire technique et prescripteur - permet d'éclairer l'évolution industrielle de l'audiovisuel et l'apparition de nouveaux business models. Les positionnements stratégiques des chaînes - en termes de ciblage, programmation, business model - s'appuient assez largement sur un positionnement sur la chaîne de valeur, entre place de marché et prescripteur. En termes opérationnels, l’imbrication historique de ces 
deux fonctions rend parfois difficile ce choix de positionnement : les difficultés autour des modèles de télévision par internet peuvent se lire comme les atermoiements des opérateurs à opter pour un modèle, entre le push (la TV) et le pull (l'internet).

De manière plus générale, que nous apprend l'exemple de l'audiovisuel sur les notions d'intermédiation et de prescription ? D’abord qu'elles sont étroitement liées et que chaque niveau d'intermédiation implique un processus de sélection qui restreint l'offre : par nature, l'intermédiation implique la prescription. L’offre, dès lors qu'elle implique une intermédiation avec le consommateur, se structure autour de pôles de prescription, dont la valeur réside dans la capacité à offrir une variété et une exclusivité. Cette capacité fait la valeur de la marque intermédiaire, par exemple des marques de grande distribution. La distribution (en tant que place de marché) est aussi prescription. On peut donc parler de marché à prescripteur, de marché de la prescription, mais il faut aussi parler de marché prescripteur.

Les deux modèles que nous avons chercher à caractériser se retrouvent également dans d'autres secteurs économiques. Le médecin est sans nul doute un prescripteur unique. Son diagnostic effectué, il impose au patient des médicaments, sans lui laisser le choix entre telle et telle marque. Du point de vue du consommateur, il n’y a qu'une décision à prendre : le choix du médecin imposera le choix du traitement. Les distributeurs du secteur de l'habillement relèvent du modèle multiple : ils sélectionnent un certain nombre de produits et de marques et les proposent aux consommateurs. Dans ce cas, la concurrence s'établit progressivement davantage entre les réseaux de distribution et de boutiques de marque qu'entre les produits (cf . (Abecassis 1998)

La réciproque est-elle vraie? Si tout intermédiaire, toute place de marché est prescription, tout prescripteur est-il place de marché ? La réponse est oui si l'on considère que la dimension importante du marché n’est pas la disponibilité physique des produits mais leur composante informationnelle. C’est l'un des enseignements du commerce électronique d'avoir montré qu'une place de marché pouvait être virtuelle, en se réduisant essentiellement à la fonction d'information, de comparaison et de choix entre des produits. Cela nous a conduits, dans notre modèle de la prescription, à dissocier les fonction 1) de «place de marché » (assurant la présentation de l’offre à la demande), 2) d'organisation de la rencontre proprement dite entre l'offre et de la demande (aide au choix et matching) et 3) de la délivrance des produits et services fournis au consommateur final (composante logistique, distribution physique, intermédiation technique). L'analyse que l'on peut tirer de la formalisation effectuée à partir du cas de l'audiovisuel montre que les deux premières dimensions (présentation de l'offre et aide au choix) peuvent cependant être comprises comme deux types-idéaux (prescription unique ou prescription multiple) d’une même fonction (marché-prescription). 


\section{Bibliographie}

Abecassis, C. (1998). Technologies de l'information et filière : dimensions clés et idéaux-types à partir du cas de l'habillement. Paris, Ecole polytechnique.

Bailey, J. P. (1998). Intermediation and Electronic Markets :Aggregation and Pricing in Internet Commerce. Technology, Management and Policy. Cambridge (Mass.), M.I.T.

Bailey, J. P. and L. W. McKnight, Eds. (1997). Internet Economics. Cambridge (Mass.), MIT Press.

Benghozi, P.-J. (2001). "Relations interentreprises et nouveaux modèles d'affaires." Revue Economique 52(Hors Série "Economie de l'Internet"): 167-190.

Benghozi, P.-J. and T. Paris (2000). "L'Industrie de la musique à l'âge d'internet : nouveaux enjeux, nouveaux modèles, nouvelles stratégies." Gestion 2000 Avril - Juin 2001.

Betancourt, R. R. and D. A. Gautschi (1993). "Two Essential Characteristics of Retail Markets and their Economic Consequences." Journal of Economic Behavior and Organization 21(3): 277-94.

Brousseau, E. (1999). The Governance of Transaction by Commercial Intermediaries : An Analysis of the Reengineering of Intermediation by Electronic Commerce. Third Conference of the International Society for New Institutional Economics, Washington DC, USA.

Brynjolfsson, E. and B. Kahin (2000). Understanding the Digital Economy, Data, Tools and Researchs. Cambridge (Mass.), MIT Press.

Brynjolfsson, E. and M. D. Smith (1999). Frictionless Commerce ? A Comparison of Internet and Conventional Retailers. Understanding the Digital Economy Data, Tools and Research, Washington D.C., US Department of Commerce.

Buzzel, R. (1985). Marketing in the Electronic Age. Boston (Mass.), Harvard Business School Press.

Commons, J. R. (1934). Institutional Economics. Its Place in Political Economy, The MacMillan Company.

Dang_Nguyen, G. (2001). L'entreprise numérique. Paris, Economica.

Diberder, A. L. and N. Coste-Cerdan (1986). La télévision. Paris, La Découverte.

Farris, P., J. Oliver, et al. (1989). "The relationship Between Distribution and Market Share." Marketing Science 8(2): 107-27.

Gensollen, M. (1999). "La création de valeur sur internet." éseaux 97: 15-76. 
Gille, L. and P. Mathonet (1994). L'intermédiation électronique. Paris, Commissariat Général du Plan.

Guillou, B. and J.-G. Padioleau (1988). La régulation de la télévision. Paris, La documentation française.

Hackett, H. C. (1992). "A Comparative Analysis of Merchant and Broker Intermediation." Journal of Economic Behavior and Organization 18(3): 299-315.

Hagel, J. I. and W. J. Lansing (1994). "Who owns the Customer." The Mc Kinsey Quaterly(4).

Hatchuel, A. (1995). Les marchés à prescripteurs :Crises de l'échange et genèse sociale. L'inscription sociale du marché. A. Jacob and H. Vérin. Paris, L'Harmattan.

Hatchuel, A. (2001). Quel horizon pour les sciences de gestion ? Vers une théorie de l'action collective. Les nouvelles fondations des sciences de gestion. A. David, A. Hatchuel and R. Laufer. Paris, Vuibert.

Jensen, M. C. and W. H. Meckling (1976). "Theory of the Firm, Managerial Behavior, Agency Costs and Ownership Structure." Journal of Financial Economics: 305-360.

Kollock, P. (1999). The Production of Trust in Online Markets. Advances in Group Processes. M. M. E.J. Lawler, S. Thyne, H.A. Walker. Greenwich, CT, JAI Press.

Lendrevie, J. and D. Lindon (2000). Mercator. Paris, Dalloz.

Lewis, M. K., Ed. (1995). Financial Intermediaries. International Library of Critical Writings in Economics. Aldershot U.K., Elgar.

Michel, D., R. Salle, et al. (1996). Marketing industriel : stratégies et mise en oeuvre. Paris, Economica.

Myerson, R. B. and M. A. Satterthwaite (1983). "Efficient Mechanisms for Bilateral Trading." Journal of Economic Theory 29(2): 265-81.

Scherer, F. M. and D. Ross (1990). Industrial Market Structure and Economic Performance. Boston, Houghton Mifflin.

Simon, J.-P. (1991). L'Esprit des règles, réseaux et réglementation aux Etats-Unis. Paris, L'Harmattan.

Spulber, D. F. (1996). "Market Microstructure and Intermediation." Journal of Economic Perspectives 10(3): 135-152.

Stern, L. W. and F. Sturdivant (1987). "Customer-Driven Distribution Channels." Harvard Business Review 65(4): 34-41.

Tapscott, D. (1999). Creating Value on the Network Economy, Harvard Business Review Book Series.

Zwass, V. (1998). Structure and Macro Level Impacts of Electronic Commerce : From Technological Infrastructure to Electronic Marketplaces. Emerging Information Technologies. K. E. Kendall. Thousand Oaks, CA, Sage Publications. 